\title{
Inline-Analyse der Dispergiergüte von Kunststoff- Compounds
}

\author{
S. Wöckel, H. Arndt, U. Steinmann, \\ Institut für Automation und Kommunikation (ifak) e.V., Magdeburg, Deutschland
}

\section{Kurzfassung}

Ein methodisch neuer statistischer Ansatz zur Charakterisierung der Feststoffphase in Polymerschmelzen anhand der Streuung von Ultraschallwellen wird vorgestellt. Gegenüber bekannten Transmissionsverfahren ermöglicht die Messung in Rückstreuung die Analyse höher konzentrierter Stoffsysteme. Die statistische Betrachtung der Streuamplitude und deren Verringerung in Abhängigkeit der Zeit liefern Informationen zur Partikelkonzentration- und größenverteilung. Hierdurch bietet sich eine verfahrenstechnische Alternative zu aufwändiger Offline-Analytik.

Schlagwörter: Polymerschmelze, Partikelcharakterisierung, Ultraschall, Streuverfahren.

\section{Motivation}

In der Kunststoffindustrie sind InlineMessverfahren gefordert, die es erlauben, kontinuierlich die Produktqualität zu überwachen und die Effizienz des Prozesses zu steigern. Die meisten Kunststoffe bestehen aus einem Basispolymer und zusätzlich eingebrachten Füllstoffen bzw. Additiven, welche gezielt z. B. die mechanische Eigenschaften, Leitfähigkeit, Entflammbarkeit oder Farbe des Materials beeinflussen. Diese werden als Compounds bezeichnet und bei den nachfolgenden formgebenden Prozessen zum Halbzeug- oder Endprodukt verarbeitet. Bei der Kunststoffaufbereitung wird das Basispolymer aufgeschmolzen und in einem Extruder mit den Feststoffpartikeln der Additive und Füllstoffe vermischt (vgl. Bild 1). Typische Kunststoffcompounds enthalten Additive und Füllstoffe mit Partikelgrößen bis zu einigen $100 \mu \mathrm{m}$.

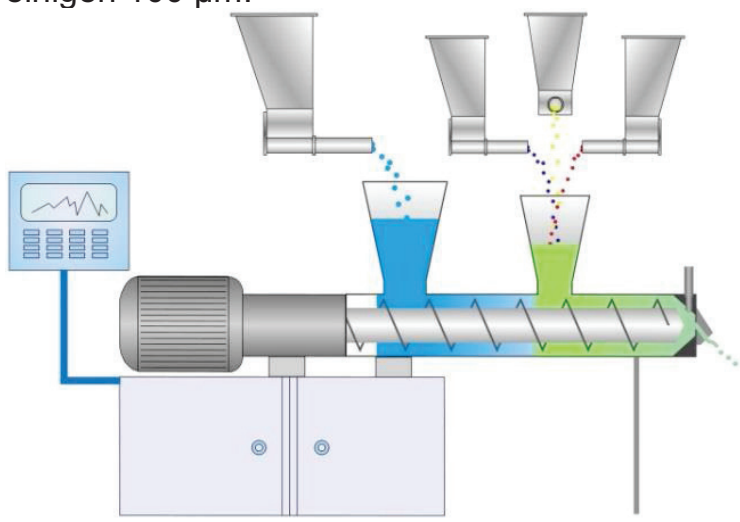

Bild 1: Schema eines Extruders: Das Polymer (blau) wird durch Scherung in der Förderschnecke aufgeschmolzen und mit Füllstoffen und Additiven (lila, gelb und rot) zum gezielten verändern der gewünschten Materialeigenschaften vermischt.
Zur Erreichung der gewünschten Materialeigenschaften ist die homogene Verteilung der Additive und Füllstoffe in der Polymermatrix entscheidend. Folglich stellt die Dispergiergüte der Polymermatrix eines der zentralen Qualitätskriterien bei der Kunststoffaufbereitung dar. Die Überwachung der Partikelverteilung der Füllstoffe ist bereits in der Polymerschmelze von wesentlicher Bedeutung, um Prozesse optimal zu regeln und Produktqualitäten gezielt zu steigern.

\section{Klassische Methoden}

Eine Analyse findet entweder zeitversetzt und dem Produktionsprozess nachgeschaltet im Labor oder parallel zum Prozess am Extruder statt. Generell werden anwendungsspezifisch unterschiedliche Messmethoden eingesetzt, welche i. d. R. arbeits- und zeitaufwändig sind, meist keine lückenlose $100 \%$-Prozesskontrolle ermöglichen und vorrangig einen integralen Wert aufweisen.

\section{Labormethoden}

Bei den Labormessungen werden vorrangig optische oder Elektronenraster-Mikroskope eingesetzt, die meist hohe Kosten und einen zusätzlichen Probenpräparationsaufwand bedeuten. Des Weiteren bilden diese Verfahren nur einen ausgewählten Bereich des Polymers ab und erlauben keine ganzheitliche Bestimmung der Materialparameter.

Weiterhin kann die Abhängigkeit zwischen Dispergiergüte und mechanischen Eigenschaften genutzt werden [1-3]. Dazu wird z. B. über die Schlag- oder Zugfestigkeit auf die Ver- und Zerteilung der Feststoffe im Compound geschlossen. Nachteile liegen in der aufwändigen 
Probenvorbereitung, schlechten Reproduzierbarkeit und der Abhängigkeit der mechanischen Eigenschaften von weiteren Einflussgrößen.

Die bislang einzige standardirisierte Methode zur Bestimmung der Dispergiergüte stellt der Druckfiltertest nach DIN EN 13900-5 dar [4]. Diese Methode ist hauptsächlich für Pigmente $(<1 \mu \mathrm{m})$ und nicht für grobkörnige $(<100 \mu \mathrm{m})$ Füllstoffe entwickelt worden. Zudem liefert sie keine Informationen zur Menge und Größe von Agglomeraten.

\section{Prozessmessmethoden}

Einen Überblick zu den gängigen Verfahren der Prozessüberwachung in der Kunststoffaufbereitung wird von Hochrein und Alig $[5,6]$ gegeben. In den Arbeiten ist auch eine online Variante des Druckfiltertests [5] beschrieben, die über einen Bypass am Extruder realisiert wird und zeitgleich zum Prozess stattfindet. Die prinzipbedingten Nachteile des Verfahrens bleiben jedoch bestehen.

Die optische Kleinwinkellichtstreuung, die in anderen Industriebereichen als dominierende Methode zur Partikelcharakterisierung gilt, liefert einen Ansatz zur Anwendbarkeit an Dispersionen und Gemischen mit zwei polymeren Phasen [7, 8]. Diese kann jedoch nur bei transluzenten Schmelzen eingesetzt werden.

Eine weitere Inline-Methode stellt die Überwachung mit optischen CCD Zeilenkameras direkt am extrudierten Endprodukt dar. Hiermit lassen sich Fremdstoffe, Agglomerate und Inhomogenitäten detektieren, wobei die Transparenz des Polymers eine wesentliche Voraussetzung ist und somit vorrangig bei der Folienextrusion angewendet wird.

\section{Ultraschall-Methoden}

Die in anderen Industriebereichen etablierte Ultraschall (US)-Methoden basieren auf der akustischen Durchschallung der Dispersion und zeichnen sich durch eine schnelle, inlinefähige Arbeitsweise aus. Diese kann im Gegensatz zu optischen Methoden auch an hoch konzentrierten und opaken Proben ohne Verdünnung angewendet werden. Ferner ist die US-Technik auch unter industrierelevanten Prozessbedingungen anwendbar und erlaubt den Einsatz in einem weiten Konzentrations- und Partikelgrößenbereich. Hierzu werden die US-Wandler häufig in einer sich gegenüberliegenden Sender-Empfänger-Anordnung positioniert (vgl. Bild 2). Mit einer solchen Anordnung ist es möglich, sowohl den Transmissions- als auch den Extinktionsanteil (Dämpfung) der mit der Dispersion wechselwirkenden Schallwelle zu bestimmen sowie daraus Informationen zur
Dimension, Größenverteilung und Konzentration der Teilchen abzuleiten [5, 6, 9, 10]. Der gemessene effektive Dämpfungsfaktor ist im Wesentlichen das Resultat von vier Dämpfungsmechanismen: der thermischen Partikelpulsation, der visko-inertialen Oszillation, der Absorption und der Streuung an den Partikeln. Die Herausforderung besteht darin, mit adäquaten Modellen [11-18] den Streuanteil, der mit der Größe, Anzahl und Verteilung der Partikel korreliert, zu trennen. Zur Verbesserung des gewünschten Streueffekts werden typischerweise Schallwellen mit Wellenlängen kleiner der Partikelgröße verwendet (Bild 3). Je größer das Partikel im Vergleich zur Wellenlänge wird, desto höher ist die erfasste Streudämpfung. Versuchsreihen zur Extinktionsdämpfung in Polymerschmelzen in Abhängigkeit der Verteilung von Partikeln zweier Größenfraktionen $(1,4 \mu \mathrm{m}$ und $37 \mu \mathrm{m})$ zeigen (Bild 4), dass bereits auf einfache Weise mit Hilfe einer linearen Approximation das Vorhandensein bzw. der Anteil der "größeren" Partikelfraktion als Modellsystem für Agglomerate in der Schmelze überwacht werden kann.

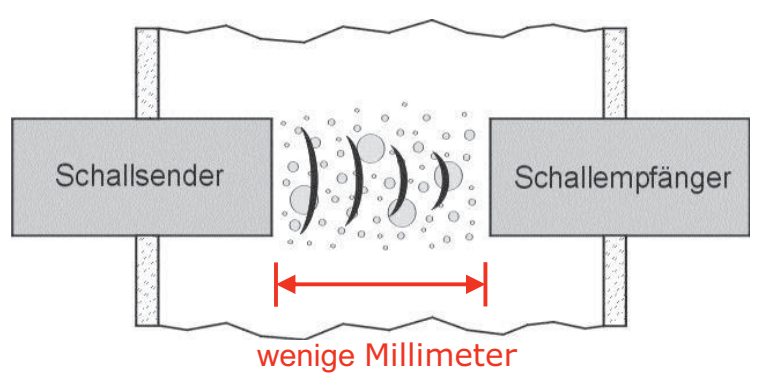

Bild 2: Schematischer Aufbau einer USTransmissionsanordnung: Sender und Empfänger liegen sich dabei direkt gegenüber.

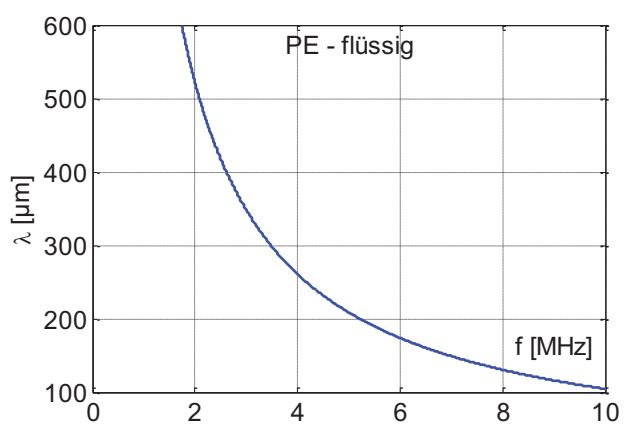

Bild 3: Wellenlänge $\lambda$ in Polyethylen (PE flüssig) in Abhängigkeit von der Anregungsfrequenz $f$. 


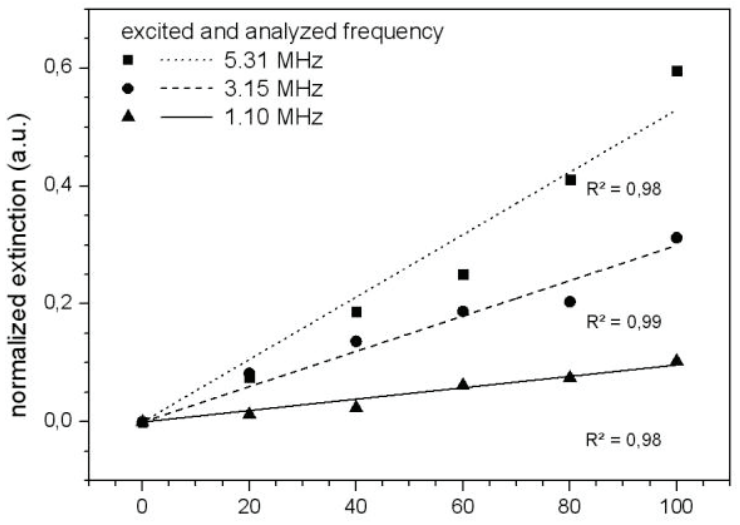

rate of calcium carbonate with a larger particle size (wt.-\%)

Bild 4: Normierter Extinktionskoeffizient von Polypropylen in Abhängigkeit des gravimetrieschen Anteils von Kreidepartikel mit $37 \mu \mathrm{m}$ Durchmesser anstelle von Partikel mit 1,4 $\mu \mathrm{m}$ Durchmesser für drei ausgewählte Frequenzen bei konstantem Füllstoffanteil von $5 \%$ (vgl. Tab. 1) [10].

Aufgrund der erheblichen Schalldämpfung ist es bei hoch konzentrierten Stoffsystemen erforderlich, den Abstand zwischen Sende- und Empfangswandler hinreichend klein zu gestalten, um ein noch auswertbares Messsignal zu erhalten. Entsprechend sind die Extinktionsverfahren meist auf Bypass-Anwendungen beschränkt und führen beim Anwender nur zu geringer Akzeptanz.

Dieser Problemstellung widmet sich das neuartige Ultraschall-Reflexionsmess-verfahren. Dabei wird anstelle der Transmission die an Füllstoffpartikeln zurückgestreute akustische Welle kontinuierlich und direkt im Kunststoffverarbeitungsprozess erfasst (vgl. Bild. 5). Anhand dieses Empfangssignals soll die disperse Phase abhängig von der Schallfrequenz sowie der Konzentration und Größe der Füllstoffpartikel analysiert werden.

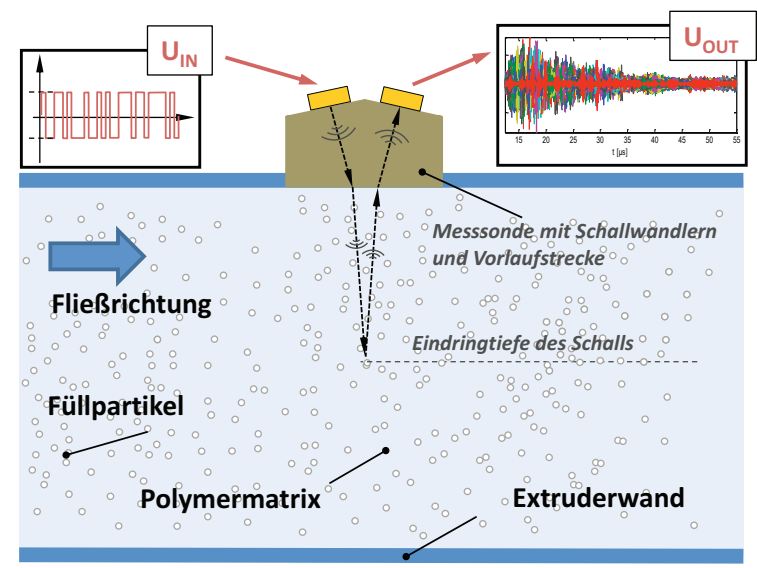

Bild 5: Prinzipskizze des Ultraschall-RückstreuMessverfahrens zur Bestimmung der Dispergiergüte in Kunststoffschmelzen
Tabelle 1: Materialparameter

\begin{tabular}{|c|c|c|}
\hline Material & $\rho\left[\mathrm{kg} / \mathrm{m}^{3}\right]$ & $C_{\text {Longitudinal }}[\mathrm{m} / \mathrm{s}]$ \\
\hline PE (flüssig) & 920 & 1045 \\
\hline Hydrocarb. 95T & 2700 & 7400 \\
\hline Spheriglass 3000CP & 1590 & 6790 \\
\hline
\end{tabular}

\section{Rückstreuung und Standardabweichung}

Zur Charakterisierung von Dispersionen und Suspensionen existieren bereits Messverfahren bzw. prozessfähige Messgeräte, die auf der Analyse der Schallstreuung an einzelnen Partikeln beruhen [UPM Ultrasonic Particle Monitor, Netherlands Organization for Applied Scientific Research TNO], [Model AS3 Acoustic Sensor, Galvanic Applied Sciences Inc.]. Den gemessenen Streusignalen werden dabei Einzelechos, d. h. von einzelnen Partikeln reflektierte Schallwellen zugeschrieben. Diese Messgeräte sind für den Einsatz in sehr gering konzentrierten Partikelsystemen konzipiert, da sie einzelne und voneinander unabhängige Streuereignisse auswerten und meist zur Einzelpartikeldetektion genutzt werden.

Kerngedanke des auf Ultraschallreflexion basierenden Verfahrens (Bild 5) zur Messung an hochdämpfenden Polymerschmelzen mit hohem Partikelanteil ist der Einsatz breitbandiger Sende-/Empfangswandler. Diese sind hinsichtlich einer hohen Sendeleistung, eines der Eindringtiefe entsprechenden Messfensters und hoher Empfangssensitivität optimal aufeinander abgestimmt. Auf diese Weise soll der von den Partikeln gestreute Schallanteil erfasst und hinsichtlich der Intensität, der Laufzeit (entspricht der Eindringtiefe) und der Schallfrequenz analysiert werden.

In der klassischen Reflexionsanordnung steht dabei der Sende-/Empfangswandler über eine Vorlaufstrecke im Kontakt mit der Dispersion. Die aktive Oberfläche der Wandler ist durch die Vorlaufstrecke aus temperaturbeständigem Kunststoff sowohl thermisch als auch abrasiv geschützt. Zum Einsatz kommen Wandler mit Mittenfrequenzen von $2 \ldots 4,5 \mathrm{MHz}$ bei einer relativen $6 \mathrm{~dB}$-Bandbreite von $60 \%$ (Olympus). Die Anregung erfolgt konventionell als Impuls (US-Pulser/Receiver UT340, Utex Scientific Instruments, Inc.) oder kodiertes Signal [19].

Das nachfolgend beschriebene Verfahren setzt voraus, dass sich die Verteilung der Partikel (mit statistisch zufälliger Bewegung) innerhalb des Schallfeldes und damit die Streucharakteristik zwischen zwei aufgenommenen Einzelsignalen ändern. Dies ist i. d. R. beim Compoundierprozess durch den Massestrom gege- 
ben. Letzteres bestimmt u. a. die maximal zulässige Messgeschwindigkeit.

Die mit dem US-Wandler (2...4,5 MHz) emittierten Schallwellen haben im flüssigen Polymer (z. B. PE), wie auch annähernd in der Dispersion, eine Wellenlänge von $\lambda=250 \ldots 500 \mu \mathrm{m}$. Die Partikelgröße der Füllstoffe liegt mit $d=1 \ldots 100 \mu \mathrm{m}$ eine Größenordnung unterhalb der Wellenlänge. Entsprechend schwach ausgeprägt ist die Schallstreuung durch die Partikel [12]. Die Untersuchungen zeigen, dass selbst bei schwacher Streuung die messtechnische Erfassung und Auswertung von US-Reflexionsbzw. Rückstreusignalen möglich ist.

Die direkte Ultraschallrückstreumessung liefert nach Anregung des Schallwandlers ein Echosignal (Amplitude $p$ in Abhängigkeit von der Laufzeit $t$ ), das neben stationären Reflexionen ebenfalls die Rückstreusignale der Partikel enthält.

Ein solcher Signalverlauf ist am Beispiel einer Dispersion aus Wasser mit $\mathrm{SiO}_{2}$-Partikeln (Dichte: $2,35 \mathrm{~g} / \mathrm{cm}^{3}$, mittlere Partikelgröße: $3 \mu \mathrm{m}$ ) in Bild 6, oben gezeigt. Aus dem Echobzw. Rückstreusignal wird deutlich, dass die durch Partikel erzeugten Signalkomponenten aufgrund ihrer geringen Amplitude nur unzureichend von Signalanteilen infolge der (Mehrfach-)Reflexion und sekundären Ausbreitungsmoden in der Wellenleitervorlaufstrecke zu unterscheiden sind. Daher wurde anstelle der absoluten Auswertung der Echoamplitude deren Standardabweichung in Abhängigkeit von der Laufzeit herangezogen. Nach Erfassung von $N$ Einzelmessungen (vollständiges Echosignal infolge eines ausgesendeten Impulses) wird zu jeder Laufzeit $t$ die Standardabweichung der jeweils $N$ Amplitudenwerte bestimmt. Statische Signalanteile würden hier keinen Anteil zur Standardabweichung liefern. Demgegenüber erzeugen die zeitlich veränderlichen Partikelsignale infolge der Bewegung der Partikel durch das Messvolumen eine deutliche Standardabweichung (Bild 6, unten). Diese zeitabhängige Standardabweichung korreliert mit der Wahrscheinlichkeit der Messbarkeit der Streuung an einem Einzelpartikel zu einem bestimmten Zeitpunkt. Folglich repräsentiert eine hinreichende Anzahl von $N$ Einzelmessungen die integrale homogene Verteilung des bewegten Partikelsystems. Mittels der statistischen Verarbeitung von mehreren Messsignalen lassen sich zudem das effektive Signal-zuRausch-Verhältnis und damit die Eindringtiefe verbessern. Es wird ferner auch vorausgesetzt, dass die Bewegungsdynamik des Partikelsystems wesentlich kleiner als die Ausbreitungsgeschwindigkeit der akustischen Welle ist.
Beim Durchlaufen der Dispersion werden die emittierte und die an den Partikeln gestreuten Schallwellen in Abhängigkeit von der zurückgelegten Wegstrecke gedämpft. Die vom Schallwandler detektierbare Echosignalamplitude verringert sich demnach mit zunehmender Laufzeit - gleiches gilt für die Standardabweichung. Die Schallamplitude fällt dabei exponentiell mit der durchschallten Wegstrecke bzw. der Laufzeit ab und wird $u$. a. von der Partikelgröße und -konzentration bestimmt [20, 21].
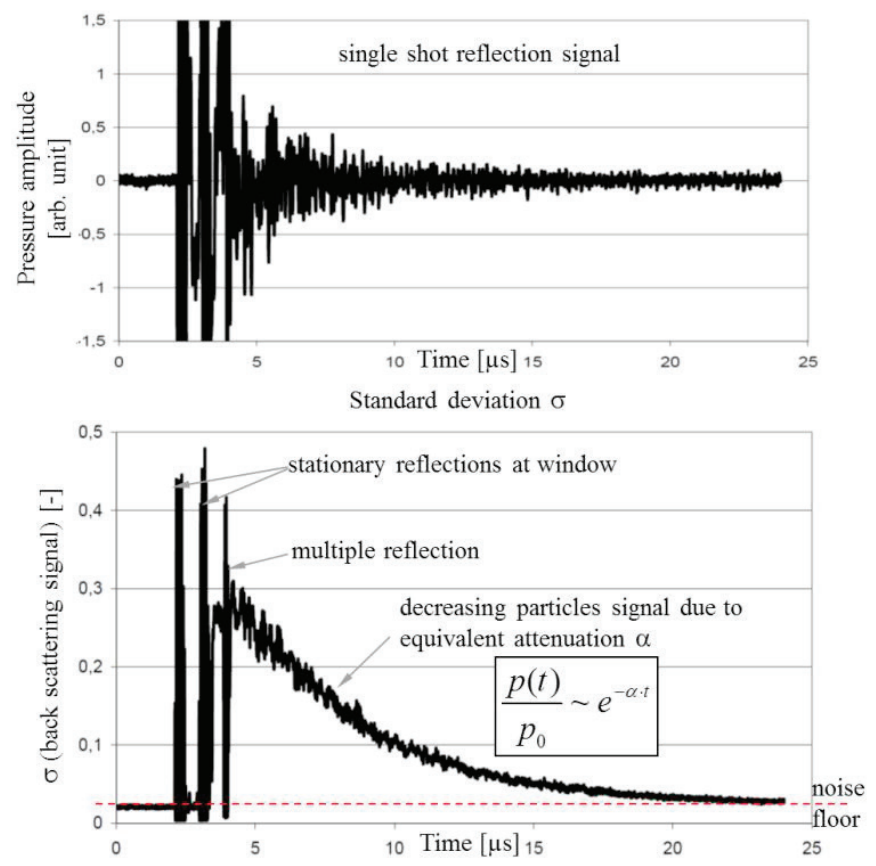

Bild 6: US-Einzel-Rückstreusignal (oben) und laufzeitabhängige Standardabweichung $\sigma$ (unten) aus $N$ $=200$ Einzelmessungen für eine Dispersion in Wasser mit $\mathrm{SiO}_{2}$ Partikeln (Partikelgröße $d<20 \mu \mathrm{m}$; $\rho=2.35 \mathrm{~g} / \mathrm{cm}^{3}$; Konzentration $\left.30 \mathrm{Gew} . .-\%\right)$.

Bei logarithmischer Skalierung der Standardabweichung kann der in Bild 6 gezeigte Verlauf abschnittsweise durch eine lineare Funktion approximiert werden:

$$
\sigma(t)[d B]=-\Delta \sigma \cdot t[\mu s]+\sigma_{\max }
$$

Die Standardabweichung $\sigma$ liefert damit einen einfachen Kennwert, dessen Amplitude (bzw. Maximum $\sigma_{\max }$ u.a. von der Streuamplitude (d.h. Partikelgröße) und der Abfall der Kurve $\Delta \sigma$ von der Anzahl der Mehrfachstreuungen (d.h. Konzentration) abhängen.

\section{Zusammenfassung}

Der Beitrag stellt die Kombination der Ultraschall-Reflexions-Methode mit einer statistischen Signalauswertung zur Charakterisierung der Dispergiergüte von Füllstoffen in Kunststoffschmelzen vor. Das Verfahren basiert auf der Erfassung der Streuung an bewegten Partikeln bei hohen Konzentrationen oder Medien 
hoher Grunddämpfung. Anhand der linearen Approximation der Standardabweichung der zeitabhängigen Amplitude der Reflexionssignale lassen sich zwei Kennwerte $\left(\sigma_{\max }\right.$ - Streuleistungsäquivalent und $\Delta \sigma$ - Dämpfungsäquivalent) ableiten. Diese repräsentieren den Einfluss der Partikel auf die Streudämpfung und liefern ähnliche Informationen wie die Extinktionsdämpfung konventioneller Transmissionsverfahren. Die statistische Verarbeitung mehrerer Signale liefert zudem ein homogenes Abbild des bewegten Partikelsystems.

Mit der Reduktion auf eine Messsonde entsteht ein einfacher Messaufbau, der nur den einseitigen Zugang zum Schmelzekanal am Extruder erfordert und die Probleme bekannter Transmissionsanordnungen umgeht.

\section{References}

[1] M. Bories, M. A. Huneault, Effect of Twin-Screw Extruder Design and Process Conditions on UItrafine $\mathrm{CaCO}_{3}$ Dispersion into PP, Int. Polymer Processing Journal 14, 1999, 3, 234.

[2] J. Tatibouët, M. A. Huneault, In-line Ultrasonic Monitoring of Filler Dispersion during Extrusion, Int. Polymer Processing J. 17, 2002, 1, 49.

[3] N. Krumbholz, T. Hochrein, et.al, Degree of dispersion of polymeric compounds determined with terahertz time-domain spectroscopy, Polymer Engineering Science 2011, 51, 109.

[4] DIN EN 13900-5:2005-07: Pigments and extenders - Methods of dispersion and assessment of dispersibility in plastics - Part 5: Determination by filter pressure value test, 2005 .

[5] T. Hochrein, I. Alig, Prozessmesstechnik in der Kunststoffaufbereitung, Vogel-Verlag, Würzburg, 2011.

[6] I. Alig, B. Steinhoff, D. Lellinger, Monitoring of polymer melt processing, Measurement Science and Technology 2010, 21, 1.

[7] B. Steinhoff, D. Lellinger, P. Pötschke, I. Alig, In-line Monitoring of the Dispersion of Nanofillers in Polymer Composites using Vibration Spectroscopy, Ultrasonic Measurement, In-line Rheology and Dielectric Spectroscopy, Proceeding Polymer Processing Society 21, 2005.

[8] S. Li, K.B. Migler, et.al, Light-scattering photometer with optical microsope for the in-line study of polymer extrusion, Journal of Polymer Science Part B 1997, 35, 2282.

[9] I. Alig, D. Lellinger, Frequency-dependence of ultrasonic velocity and attenuation in 2-phase composite systems with spherical scatterers, Journal of Applied Physics 1992, 72, 5565.

[10] G. Schober, P. Heidemeyer, et.al, Degree of dispersion monitoring by ultrasonic transmission technique and excitation of the transducer's harmonics Proceeding Polymer Processing Society 29, 2013.
Der Versuchsaufbau am Extruder und entsprechende Messergebnisse zum beschriebenen Verfahren werden im Rahmen der Posterpräsentation auf dem Dresdner Sensor Symposium gezeigt.

\section{Förderhinweis}

Das IGF-Vorhaben 18220 BG der Forschungsvereinigung Fördergemeinschaft für das SKZ e. V. wird über die AiF im Rahmen des Programms zur Förderung der industriellen Gemeinschaftsforschung und -entwicklung (IGF) vom Bundesministerium für Wirtschaft und Energie aufgrund eines Beschlusses des Deutschen Bundestags gefördert. Wir bedanken uns für die finanzielle Unterstützung.

[11] R.E. Challis, M.J.W. Povey,M.L. Mather, A.K. Holmes, Ultrasound techniques for characterizing colloidal dispersions, Rep. Prog. Phys. 68 (2005) 1541-1637.

[12] A.S. Dukhin, P.J. Goetz, Ultrasound for Characterizing Colloids, Elsevier, 2002.

[13] S.E. Kruger, et.al., Broadband ultrasonic backscattering applied to nondestructive characterization of materials, IEEE Trans. Ultrason. Ferroelectr. Freq. Control 51, 2004, 831-837.

[14] A.K. Hipp, et.al, Acoustic characterization of concentrated suspensions and emulsions. 1. Model analysis, Langmuir 18, 2002, 391-404.

[15] P.S. Epstein, R.R. Carhart, The absorption of sound in suspensions and emulsions. I. Water fog in air, J. A. S. A. 25, 1953, 553-565.

[16] J.R. Allegra, S.A. Hawley, Attenuation of sound in suspensions and emulsions: theory and experiments, J. A. S. A. 51, 1972, 1545-1564.

[12] J.J. Faran, Sound scattering by solid cylinders and spheres, J. A. S. A. 23, 1951, 405-418.

[26] A.E. Hay, D.G. Mercer, On the theory of sound scattering and viscous absorption in aqueous suspensions at medium and short wavelengths, J. A. S. A. 78, 1985, 1761-1771.

[18] A. Richter, Ultraschalldaempfungsspektroskopie grobdisperser Systeme, (PhD Thesis) Dresden University of Technology, 2008.

[19] H. Alrutz, Über die Anwendung von Pseudorauschfolgen zur Messung an linearen Übertragungssystemen, Dissertation, Georg-August Universität Göttingen, 1983

[20] R. Weser, S. Wöckel, et.al, J. Auge, Particle characterization in highly concentrated suspensions by ultrasound scatteringmethod, Sensors Actuators A 202, 2013, 30-36.

[21] R. Weser, S. Wöckel, et.al, Ultrasonic backscattering method for in-situ characterisation of concentrated dispersions, Powder Technology, Volume 268, 2014, S.177-190, ISSN 0032-5910 